\title{
Three Species Epidemiological Model with Holling Type Functional Responses
}

\author{
S Hariprasad ${ }^{1}$, M A S Srinivas ${ }^{2}$, N.PhaniKumar ${ }^{3}$ \\ ${ }^{1}$ Department of Mathematics, Anurag group of Institutions, Hyderabad, Telangana, India. \\ E-mail: srinadhunihariprasd@gmail.com \\ ${ }^{2}$ Department of Mathematics, Jawaharlal Nehru Technological University, Telangana, India. \\ E-mail: massrinivas@gmail.com \\ ${ }^{3}$ Department of Mathematics, Vignan Institute of Technology and Science, Telangana, India. \\ E-mail: nphanikumar20@gmail.com
}

Received: May 9, 2020. Revised: June 11, 2020. Accepted: July 6, 2020. Published: July 20, 2020.

Abstract: In the analytical and numerical study, the interaction between three species is modeled, where in the three species are identified as a prey, which is susceptible, the infected prey and the predator with type-II and type-Iv functional responses, which represents a mathematical model of eco-epidemiology. This study is carried out in both analytically and numerically. The boundedness of the model is studied and the stability analysis of the model is carried out at the positive equilibrium point in terms of locally and globally. The conditions for the occurrence of Hopf bifurcation with fixed biological parameter values are investigated and also it is noticed that the bifurcation occurs by sensitive changes in the parameter values of $l_{3}, l_{0}$ , and $\gamma$ which represents the growth rate of a predator, transmission rate from infected prey to susceptible prey and half-saturation constant of predator respectively. Further, the stochastic nature of the model is analyzed both analytically and numerically. It is observed that the system exhibits chaotic behavior with the sensitive parameter values which causes large environmental fluctuations.

Key words: - Infected, Susceptible, Boundedness, Local stability, Global stability, Routh-Hurwitze criteria, Hopf bifurcation, stochastic.

\section{INTRODUCTION}

Ecological systems in general dynamic, complex and non-linear in nature. The study of the Pre-Predator dynamics is one of the important area in mathematical Ecology and this study can optimized through the formulation and analysis respective Mathematical models. Many researchers like Hastigs and Powell et. all [2,3,4,5,9.10,11,12,19] examined the complex non-linear behavior of three species continues time ecological models.

In recent years many researchers focus is on the topic called Eco-Epidemiology which is the combination of 
Ecology and Epidemiology. Eco-epidemiological systems describe the spread of infectious diseases among the interacting species when at least one of the species population have infectious disease. The analysis of these systems become significant in controlling the spread of these diseases and got a lot of consideration since the KermacMckendric SIR model was proposed. In eco-epidemiology, researchers study ecological systems when the environment is polluted with infectious diseases or when either prey, predator or in both populations spread the disease. Many researchers Anderson, May, Chattopadhyay, Arino and Bate $[15,22,24]$ proposed different situations like disease in the Prey, disease in both Prey and Predator. Bate A.M. and Hilker. FM $[28,32]$ observed that predator, prey oscillations can shift when disease become endemic.

Many researchers observed that the environmental fluctuations also caused the different behaviors of the dynamic systems .J.Ripa [21] studied the effect of environmental noise in ecological food webs. R.M.May [20] investigated that the population has deviated more from steady states in a biological system involved in stochastic fluctuations by considering white noise for a population. The work of many researches $[30,32]$ in this area motivated us to compute the behavior of the coexistence state of the system having random environmental fluctuations due to white noise.

The complexity of the ecological model is considered in terms of the functional responses involved in the mathematical models. when prey/predator interacting with each other the change can occur in their density. This can be referred as functional response by Holling type-I,II and typeIII. Generally, Holling type-I,II and type-III responses have been applied to many theoretical studies. Huang and Xiao $[8,6]$ and many other researchers $[7,10,13,17,23,25,26,29,34$ ] investigated the bifurcation analysis and stability of a PreyPredator model with Type-II \& Type-IV responses.

Here the converted infected prey to susceptible will not be infected again which is the assumption we consider in this model. The predator will have interaction either with infected prey or susceptible prey, not both at a time.

\section{MATHEMATICAL MODEL}

The proposed model is

$$
\begin{aligned}
& \frac{d x}{d t}=a x-b x^{2}-\frac{l_{0} x y}{\alpha+x}-\frac{l_{1} x z}{\beta+x^{2}}+\delta y \\
& \frac{d y}{d t}=\frac{l_{0} x y}{\alpha+x}+\frac{l_{2} y z}{\gamma+y}-\delta y \\
& \frac{d z}{d t}=\frac{l_{3} x z}{\beta+x^{2}}-\frac{l_{2} y z}{\gamma+y}-d_{2} z
\end{aligned}
$$

Here ' $x$ ' is the susceptible prey density, ' $y$ ' is the infected prey density and ' $z$ ' is the Predator density at any instant of time $t$. The parameter ' $a$ ' is growth rate of susceptible prey; the parameter ' $b$ ' is intraspecific competition among individuals of prey $\mathrm{x}$; the parameters $\alpha, \beta, \gamma$ are half saturation constants $; l_{0}, l_{2}$ are rate of infection; $l_{1}, l_{3}$ are the maximal growth rate of the species ; $\delta$ is the rate of infected prey individuals to recover and reenter into susceptible prey; $d_{2}$ is mortality rate of the predator, it is evident that all parameters are positive.

The system (2.1) has eleven parameters. It is evident that dealing a system having more number of parameters is challenging and required more complicated analysis, reformulating a model in dimensionless type is helpful from many aspects. This procedure will facilitate to observe the consistency of the model equations and ensure that each term have an equivalent set of units in equation. non-dimensionalizing the model reduces the number of free parameters and divulges a smaller set of quantities that govern the dynamics of model. Consider the model values

$\alpha_{1}=\frac{a \alpha}{l_{0} \gamma} ; \alpha_{2}=\frac{b \alpha^{2}}{l_{0} \gamma} ; \alpha_{3}=\frac{l_{1} \gamma}{l_{2} \alpha^{2}} ; \alpha_{4}=\frac{\delta}{l_{0}} ; \alpha_{5}=\frac{\alpha}{\gamma} ; \alpha_{6}=\frac{\delta \alpha}{l_{0} \gamma} ; \alpha_{7}=\frac{l_{3}}{l_{0} \gamma}$

$$
\alpha_{8}=\frac{l_{2} \alpha}{l_{0} \gamma} ; \alpha_{9}=\frac{d_{2} \alpha}{l_{0} \gamma} ; k_{1}=\frac{b \alpha}{a} ; k_{2}=\frac{\delta}{l_{0}} ; k_{3}=\frac{d_{2} \alpha}{l_{3}} ; m_{0}=\frac{\beta}{\alpha^{2}} .
$$

After non-dimensionalization, The proposed model (1.1 ) becomes 


$$
\begin{aligned}
& \frac{d x}{d t}=\alpha_{1} x\left(1-k_{1} x\right)-\frac{x y}{1+x}-\alpha_{3} \frac{x z}{m_{0}+x^{2}}+\alpha_{4} y \\
& \frac{d y}{d t}=\alpha_{5} y\left(\frac{x}{1+x}-k_{2}\right)+\frac{y z}{1+y} \\
& \frac{d z}{d t}=\alpha_{7} z\left(\frac{x}{m_{0}+x^{2}}-k_{3}\right)-\alpha_{8} \frac{y z}{1+y}
\end{aligned}
$$

\section{THE BOUNDEDNES OF THE SYSTEM}

In this section, we will attain some adequate conditions for the boundedness of (2.2).

Theorem(3.1): The system (2.2) is uniformly bounded.

Proof: we consider a function $\Phi(t)=x+y+z$, , then

$$
\begin{gathered}
\frac{d \Phi}{d t}=\frac{d x}{d t}+\frac{d y}{d t}+\frac{d z}{d t} \\
\frac{d \Phi}{d t}=\alpha_{1} x\left(1-k_{1} x\right)-\frac{x y}{1+x}\left(1-\alpha_{5}\right)-\frac{x z}{m_{0}+x^{2}}\left(\alpha_{3}-\alpha_{7}\right) \\
\quad-\frac{y z}{1+y}\left(\alpha_{8}-1\right)+\alpha_{4} y-\alpha_{5} y k_{2}-\alpha_{7} z k_{2}
\end{gathered}
$$

Choose $\alpha_{5}<1, \alpha_{8}>1$, and $\alpha_{3}>\alpha_{7}$, then

$$
\frac{d \Phi}{d t} \leq \alpha_{1} x\left(1-k_{1} x\right)+\alpha_{4} y-\alpha_{5} y k_{2}-\alpha_{7} z k_{2}
$$

Now we choose arbitrary positive real number $\rho$ for which

$$
\begin{aligned}
\frac{d \Phi(t)}{d t}+\rho & \Phi(t) \leq x\left(\alpha_{1}-\alpha_{1} k_{1} x+\rho\right)-y\left(\alpha_{5} k_{2}-\alpha_{4}-\rho\right) \text { holds. } \\
& -z\left(\alpha_{7} k_{2}-\rho\right)
\end{aligned}
$$

For simplicity we take

$$
\begin{aligned}
& 0<\rho \leq \min \left(\alpha_{5} k_{2}-\alpha_{4}, \alpha_{7} k_{2}\right), \text { Therefore } \\
& \frac{d \Phi(t)}{d t}+\rho \Phi(t) \leq x\left(\alpha_{1}-\alpha_{1} k_{1} x+\rho\right) \leq \frac{\left(\alpha_{1}+\rho\right)^{2}}{4 \alpha_{1} k_{1}}=\mathrm{H} .
\end{aligned}
$$

Here $\mathrm{H}$ is the maximum value of $x\left(\alpha_{1}-\alpha_{1} k_{1} x+\rho\right)$. So,

$$
\frac{d \Phi(t)}{d t}+\rho \Phi(t) \leq \mathrm{H}
$$

Then we obtain $\Phi(t) \leq \frac{\mathrm{H}}{\rho}+\left(\Phi(0)-\frac{\mathrm{H}}{\rho}\right) e^{-\rho t}$ for $t \geq 0$. As $\underset{(2.2)}{t \rightarrow \infty}$, then $\Phi(t) \leq \frac{\mathrm{H}}{\rho}$. Thus $\Phi(t)$ lies between 0 and $\frac{\mathrm{H}}{\rho}$, Therefore, $\Phi(t)$ is bounded in $R_{+}^{3}$.

\section{STEADY STATES}

The system has the following five steady state solutions resulting from $\frac{d x}{d t}=0, \frac{d y}{d t}=0, \frac{d z}{d t}=0$.

1) $\left.(0,0,0) 2)\left(1 / k_{2}, 0,0\right) 3\right) P\left(x^{\bullet}, y^{\bullet}, 0\right)$, where $x^{\bullet}=\frac{k_{2}}{1-k_{2}}$, $y^{\bullet}=\frac{\left(1-k_{2}\right)^{2}}{\alpha_{1} k_{2}\left(\alpha_{4}-k_{2}\right)\left(k_{1} k_{2}+k_{2}-1\right)}$

4) $P\left(\begin{array}{c}\Lambda \\ x, 0, z\end{array}\right)$ where $x=\frac{1+\left(1-4 k_{3}^{2} m_{0}\right)^{\frac{1}{2}}}{2 k_{3}}$,

$\Lambda_{z}=\frac{\alpha_{1}\left(1-k_{1} x\right)\left(m_{0}+x^{2}\right)}{\alpha_{3}}$

5) the coexistent steady state is obtained by from the equations:

$\left\lfloor\alpha_{1}\left(1-k_{1} x\right)-\alpha_{3} \frac{z}{m_{0}+x^{2}}\right\rfloor+y\left\lfloor\alpha_{4}-\frac{x}{1+x}\right\rfloor=0$

$\left\lfloor\alpha_{5}\left(\frac{x}{1+x}-k_{2}\right)+\frac{z}{1+y}\right\rfloor=0$

$\left\lfloor\alpha_{7}\left(\frac{x}{m_{0}+x^{2}}-k_{3}\right)-\alpha_{8} \frac{y}{1+y}\right\rfloor=0$

Solving equations (3.2) \& (3.3), we get

$$
\begin{gathered}
y^{*}=\frac{\alpha_{7}\left(x^{*}-k_{3}\left(m_{0}+x^{* 2}\right)\right)}{\left[\left(\alpha_{8}+\alpha_{7} k_{3}\right)\left(m_{0}+x^{* 2}\right)-\alpha_{7} x^{*}\right]} \\
z^{*}=\frac{\alpha_{5} \alpha_{8}\left[k_{2}+x^{*}\left(k_{2}-1\right)\right]\left[m_{0}+x^{* 2}\right]}{\left(1+x^{*}\right)\left[\left(\alpha_{8}+\alpha_{7} k_{3}\right)\left(m_{0}+x^{* 2}\right)-\alpha_{7} x^{*}\right]}
\end{gathered}
$$


let $\quad y^{*}=\frac{-k_{3}\left\lfloor\left(x^{*}-\alpha^{*}\right)\left(x^{*}-\beta^{*}\right)\right\rfloor}{\left(\alpha_{8}+\alpha_{7} k_{3}\right)\left[\left(x^{*}-\gamma^{*}\right)\left(x^{*}-\delta^{*}\right)\right]}$

$z^{*}=\frac{\alpha_{5} \alpha_{8}\left[k_{2}+x^{*}\left(k_{2}-1\right)\right]\left[m_{0}+x^{* 2}\right]}{\left(1+x^{*}\right)\left(\alpha_{8}+\alpha_{7} k_{3}\right)\left[\left(x^{*}-\gamma^{*}\right)\left(x^{*}-\delta^{*}\right)\right]}$

Where $\quad \alpha^{*}=\frac{1-\sqrt{1-4 m_{0} k_{3}^{2}}}{2 k_{3}}, \beta^{*}=\frac{1+\sqrt{1-4 m_{0} k_{3}^{2}}}{2 k_{3}}$;

$$
\begin{aligned}
& \gamma^{*}=\frac{\alpha_{7}-\sqrt{\alpha_{7}^{2}-4 m_{0}\left(\alpha_{8}+\alpha_{7} k_{3}\right)^{2}}}{2\left(\alpha_{8}+\alpha_{7} k_{3}\right)}, \\
& \delta^{*}=\frac{\alpha_{7}+\sqrt{\alpha_{7}^{2}-4 m_{0}\left(\alpha_{8}+\alpha_{7} k_{3}\right)^{2}}}{2\left(\alpha_{8}+\alpha_{7} k_{3}\right)}
\end{aligned}
$$

whenever $k_{2}>1$ and $\left(\alpha^{*}<\delta^{*}<x^{*}<\beta^{*}\right)$, then the equilibrium point $\stackrel{*}{E}=E\left(x^{*}, y^{*}, z^{*}\right)$ exists.

\section{STABILITY ANALYSIS OF COEXISTENT STEADY STATE}

Theorem(5.1): The equilibrium point $E$ is stable locally when $B_{1}>0, B_{3}>0$ and $\left(B_{1} B_{2}-B_{3}\right)>0$.

Proof:- The Jacobian matrix is $\quad J\left(E^{*}\right)=\left[\begin{array}{llc}b_{11} & b_{12} & b_{13} \\ b_{21} & b_{22} & b_{23} \\ b_{31} & b_{32} & 0\end{array}\right]$ where

$$
\begin{aligned}
& b_{11}=\left(\alpha_{1}-2 \alpha_{1} k_{1} x^{*}\right)-\frac{y^{*}}{\left(1+y^{*}\right)^{2}}-\alpha_{3} z^{*}\left(m_{0}-x^{* 2}\right)\left(m_{0}+x^{* 2}\right)^{-2} ; \\
& b_{12}=\alpha_{4}-\frac{x^{*}}{1+x^{*}}, b_{13}=-\alpha_{3} \frac{x^{*}}{m_{0}+x^{* 2}} ; \\
& b_{21}=\frac{\alpha_{5} y^{*}}{\left(1+x^{*}\right)^{2}} ; b_{22}=\frac{-y^{*} z^{*}}{\left(1+x^{*}\right)^{2}} ; b_{23}=\frac{y^{*}}{1+y^{*}} ; \\
& b_{31}=\alpha_{7} z^{*}\left(m_{0}-x^{* 2}\right)\left(m_{0}+x^{* 2}\right)^{-2} ; \\
& b_{32}=\frac{-\alpha_{8} z^{*}}{\left(1+y^{*}\right)^{2}} .
\end{aligned}
$$

The characteristic equation of $J\left(E^{*}\right)$ is $\lambda^{3}+B_{1} \lambda^{2}+B_{2} \lambda+B_{3}=0$. where

and

$$
\begin{aligned}
& B_{1}=-\left(b_{11}+b_{22}\right) ; B_{2}=\left(b_{11} b_{22}-b_{32} b_{23}-b_{12} b_{21}-b_{13} b_{31}\right) ; \\
& B_{3}=\left(b_{11} b_{32} b_{23}+b_{13} b_{31} b_{22}-b_{12} b_{23} b_{31}-b_{13} b_{21} b_{32}\right) .
\end{aligned}
$$

Here

$$
B_{1}=\frac{y^{*}}{N_{2}^{2}}\left(1+z^{*}\right)+\alpha_{3} z^{*}\left(m_{0}-x^{* 2}\right)\left(m_{0}+x^{* 2}\right)^{-2}+\alpha_{1}\left(2 k_{1} x^{*}-1\right)
$$

$$
\begin{array}{r}
B_{3}=\frac{\alpha_{8} y^{*} z^{*}}{N_{2}^{5} N_{3}^{2}}\left[y^{*} N_{3}^{2}+\alpha_{3} z^{*}\left(m_{0}-x^{* 2}\right) N_{2}^{2}+\alpha_{1}\left(2 k_{1} x^{*}-1\right) N_{2}^{2} N_{3}^{2}\right] \\
+\left[\frac{\alpha_{7} y^{*} z^{*}\left(m_{0}-x^{* 2}\right)\left(x^{*}-\alpha_{4} N_{1}\right)}{N_{1} N_{2} N_{3}^{2}}\right]+ \\
\frac{\alpha_{3} x^{*} y^{*} z^{*}}{N_{1}^{2} N_{2}^{2} N_{3}^{2}}\left[\frac{\alpha_{7} z^{*}\left(m_{0}-x^{* 2}\right) N_{1}^{2}}{N_{3}}-\alpha_{5} \alpha_{8} N_{3}\right] .
\end{array}
$$

Consider, $\Delta=B_{1} B_{2}-B_{3}$

$$
\begin{aligned}
\Delta=\frac{y^{*} z^{*}}{N_{2}^{6} N_{3}^{4}}\left[F^{* 2}+F^{*} N_{3}^{2}\left(y^{*} z^{*}-N_{2} \alpha_{8}\right)\right] & \\
+ & \frac{\alpha_{5} y^{*}\left(x^{*}-N_{1} \alpha_{4}\right)\left(F^{*}+N_{3}^{2} y^{*} z^{*}\right)}{N_{1}^{3} N_{2}^{2} N_{3}^{2}} \\
+ & \frac{\alpha_{3} \alpha_{7} z^{*} x^{*}\left(m_{0}-x^{* 2}\right) F^{*}}{N_{2}^{2} N_{3}^{3}}+ \\
\frac{\alpha_{8} y^{*} z^{*}}{N_{2}^{2}} & {\left[\frac{\left(F^{*}+N_{3}^{2} y^{*} z^{*}\right)}{N_{2}^{3} N_{3}^{2}}+\frac{\alpha_{3} \alpha_{5} x^{*}}{N_{1}^{2} N_{3}}\right]+} \\
& \frac{\alpha_{7} x^{*} y^{*} z^{*}\left(m_{0}-x^{* 2}\right)}{N_{2}^{2} N_{3}^{2} N_{1}}\left[\begin{array}{l}
\alpha_{3} z^{*} N_{1} N_{3} \\
\left.-\frac{\alpha_{3} z^{*} N_{1}}{N_{3}}-\frac{\left(x^{*}-N_{1} \alpha_{4}\right) N_{2}}{x^{*}}\right]
\end{array}\right]
\end{aligned}
$$

Where

$$
\begin{aligned}
& N_{1}=\left(1+x^{*}\right) ; N_{2}=\left(1+y^{*}\right) ; N_{3}=\left(m_{0}+x^{* 2}\right) ; \\
& F^{*}=\left(\begin{array}{l}
y^{*} N_{3}^{2}+\alpha_{3} z^{*}\left(m_{0}-x^{* 2}\right) N_{2}^{2} \\
+\alpha_{1}\left(2 k_{1} x^{*}-1\right) N_{2}^{2} N_{3}^{2}
\end{array}\right) . \\
& \Delta=B_{1} B_{2}-B_{3}>0, \\
& \text { i) } \left.\left.4 k_{1}^{2} m_{0}>1 \text { ii }\right) N_{3}^{2}>1 \text { iii }\right) \alpha_{8}<\frac{N_{1} z^{*}}{N_{3}}\left(\frac{\alpha_{7} y^{*}\left(m_{0}-x^{* 2}\right)}{\alpha_{5} N_{2}}\right)^{\frac{1}{2}} .
\end{aligned}
$$$$
\text { if }
$$

By Routh-Hurwitze principle, the steady state point $\stackrel{*}{E}$ is stable locally, if $B_{1}>0, B_{3}>0$ and $\left(B_{1} B_{2}-B_{3}\right)>0$ holds.

Theorem(5.2): Along with the conditions stated in the above theorem and If 
$\alpha_{3}<\frac{\left(m_{0}+\Psi_{1}^{* 2}\right)\left(m_{0}+\Psi_{1}^{2}\right)}{\Psi_{3}^{*}\left(\Psi_{1}+\Psi_{1}^{*}\right)}\left[\frac{\alpha_{4} \Psi_{2}^{*}}{\Psi_{1} \Psi_{1}^{*}}-\frac{\Psi_{2}^{*}}{\left(1+\Psi_{1}\right)\left(1+\Psi_{1}^{*}\right)}+\alpha_{1} k_{1}\right]$

then, the steady state point $\stackrel{*}{E}$ is stable globally.

Proof: We consider a Lyapunov function $v(t)$ such that

$v(t)=n_{1}\left\lfloor\Psi_{1}-\Psi_{1}^{*}-\Psi_{1}^{*} \ln \left(\frac{\Psi_{1}}{\Psi_{1}^{*}}\right)\right\rfloor$

$+n_{2}\left[\Psi_{2}-\Psi_{2}^{*}-\Psi_{2}^{*} \ln \left(\frac{\Psi_{2}}{\Psi_{2}^{*}}\right)\right] \quad$, where

$+n_{3}\left[\Psi_{3}-\Psi_{3}^{*}-\Psi_{3}^{*} \ln \left(\frac{\Psi_{3}}{\Psi_{3}^{*}}\right)\right]$

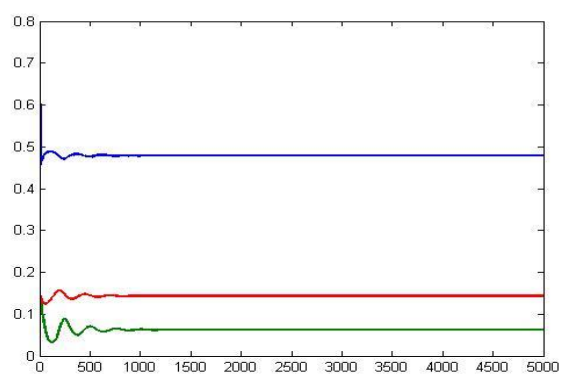

$\Psi_{1}=x, \Psi_{2}=y, \Psi_{3}=z$ and $n_{1}, n_{2}, n_{3}$ are positive constants

$\frac{d v}{d t}=n_{1}\left[\begin{array}{l}\frac{\Psi_{2}^{*}}{\left(1+\Psi_{1}\right)\left(1+\Psi_{1}^{*}\right)}+\frac{\alpha_{3} \Psi_{3}^{*}\left(\Psi_{1}+\Psi_{1}^{*}\right)}{\left(m_{0}+\Psi_{1}^{2}\right)\left(m_{0}+\Psi_{1}^{* 2}\right)} \\ -\frac{\alpha_{4} \Psi_{2}^{*}}{\Psi_{1} \Psi_{1}^{*}}-\alpha_{1} k_{1}\end{array}\right]\left(\Psi_{1}-\Psi_{1}^{*}\right)^{2}$

$-\frac{n_{2} \Psi_{3}^{*}}{\left(1+\Psi_{2}\right)\left(1+\Psi_{2}^{*}\right)}\left(\Psi_{2}-\Psi_{2}^{*}\right)^{2}$

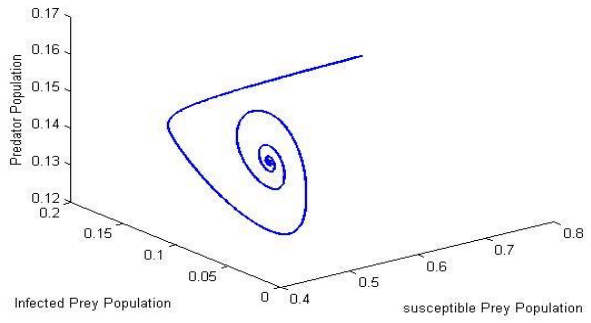

$+\left[\frac{n_{1}}{\Psi_{1}}\left(\alpha_{4}\left(1+\Psi_{1}\right)-\Psi_{1}\right)+\frac{n_{2} \alpha_{5}}{\left(1+\Psi_{1}^{*}\right)}\right] \frac{\left(\Psi_{1}-\Psi_{1}^{*}\right)\left(\Psi_{2}-\Psi_{2}^{*}\right)}{\left(1+\Psi_{1}\right)}$

(b)

$+\left[n_{3} \alpha_{7}-n_{1} \alpha_{3}-\frac{n_{3} \alpha_{7} \Psi_{1}^{*}\left(\Psi_{1}+\Psi_{1}^{*}\right)}{\left(m_{0}+\Psi_{1}^{* 2}\right)}\right] \frac{\left(\Psi_{1}-\Psi_{1}^{*}\right)\left(\Psi_{3}-\Psi_{3}^{*}\right)}{\left(m_{0}+\Psi_{1}{ }^{2}\right)}$

$+\frac{1}{1+\Psi_{2}}\left[n_{2}-n_{3} \alpha_{8}+\frac{n_{3} \alpha_{8} \Psi_{2}^{*}}{\left(1+\Psi_{2}^{*}\right)}\right]\left(\Psi_{3}-\Psi_{3}^{*}\right)\left(\Psi_{2}-\Psi_{2}^{*}\right)$.

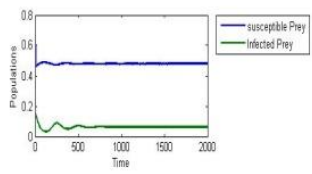

(a)

The sufficient conditions for $\frac{d v}{d t}<0$ are as follows

$$
\begin{gathered}
n_{1}=n_{2}=1, n_{3}=\frac{\left(1+\Psi_{2}^{*}\right)}{\alpha_{8}}, \alpha_{4}=\frac{\Psi_{1}\left(1+\Psi_{1}^{*}-\alpha_{5}\right)}{\left(1+\Psi_{1}^{*}\right)\left(1+\Psi_{1}\right)}, \text { and } \\
\alpha_{7}=\frac{\alpha_{3} \alpha_{8}\left(m_{0}+\Psi_{1}^{* 2}\right)}{\left(1+\Psi_{2}^{*}\right)} \\
\alpha_{3}<\frac{\left(m_{0}+\Psi_{1}^{* 2}\right)\left(m_{0}+\Psi_{1}^{2}\right)}{\Psi_{3}^{*}\left(\Psi_{1}+\Psi_{1}^{*}\right)} \\
{\left[\frac{\alpha_{4} \Psi_{2}^{*}}{\Psi_{1} \Psi_{1}^{*}}-\frac{\Psi_{2}^{*}}{\left(1+\Psi_{1}\right)\left(1+\Psi_{1}^{*}\right)}+\alpha_{1} k_{1}\right]}
\end{gathered}
$$
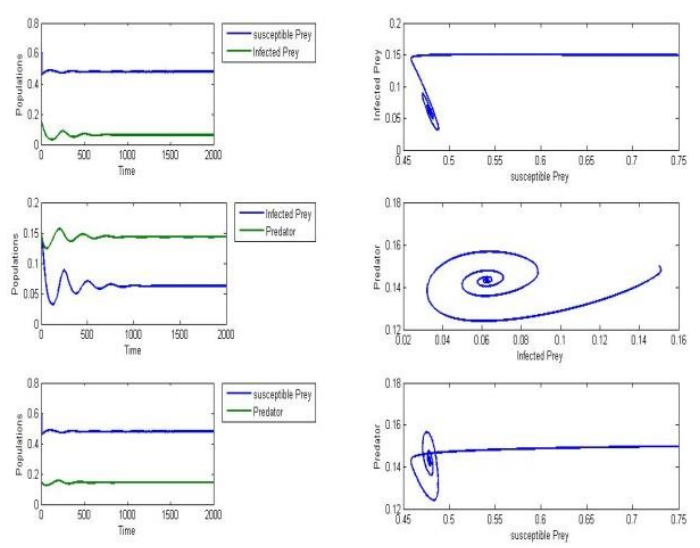

(C)

\section{Numerical Simulations:}

Figure 5.1:(a) a time profile of the steady state with respect to the populations of $\mathrm{x}, \mathrm{y}, \mathrm{z}$.

(b) phase portrait at $\stackrel{*}{E}$ $=(0.5963,0.0272,0.0001539)$ with the parameter values 
$m_{0}=0.00009982 ; \alpha_{1}=1.099912 ; \alpha_{3}=0.099872 ; \alpha_{4}=$ $0.099893 ; \alpha_{5}=0.199285 ; \alpha_{7}=0.0099 ; \alpha_{8}=0.0999389$; $k_{1}=1.92 ; k_{2}=1.00009 ; k_{3}=1.4091$.

(c) a time profile and phase portraits in two dimensional plane for the above parameter values.

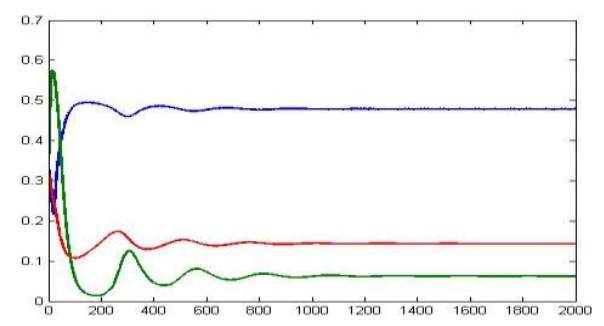

(a)

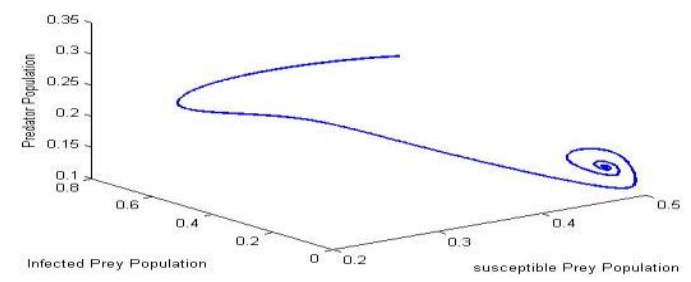

(b)
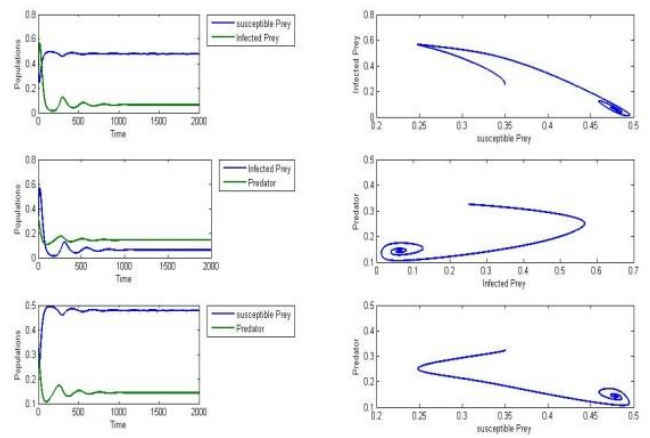

(C)
Figure 5.2:(a) a time profile of the steady state with respect to the populations of $\mathrm{x}, \mathrm{y}, \mathrm{z}$. (b) phase portrait at $\stackrel{*}{E}=(0.723$, $0.0184,0.000312)$ with the parameter values $m_{0}=0.09982$; $\alpha_{1}=1.079912 ; \alpha_{3}=0.009 ; \alpha_{4}=0.08 ; \alpha_{5}=0.2 ; \alpha_{7}=$ $0.01 ; \alpha_{8}=0.099 ; k_{1}=1.42 ; k_{2}=1.01 ; k_{3}=1.2$.

(c) a time profile and phase portraits in two dimensional plane for the above parameter values.

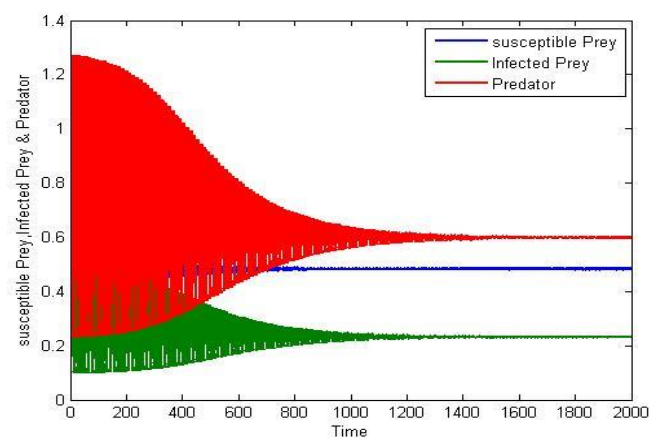

(a)

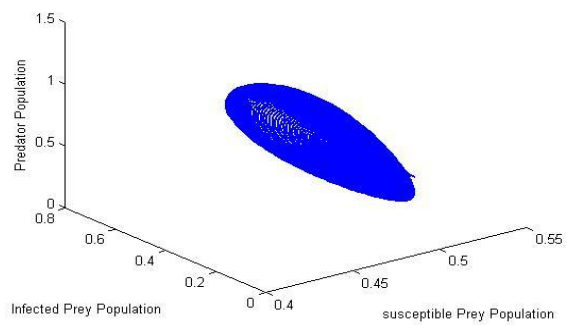

(b) 

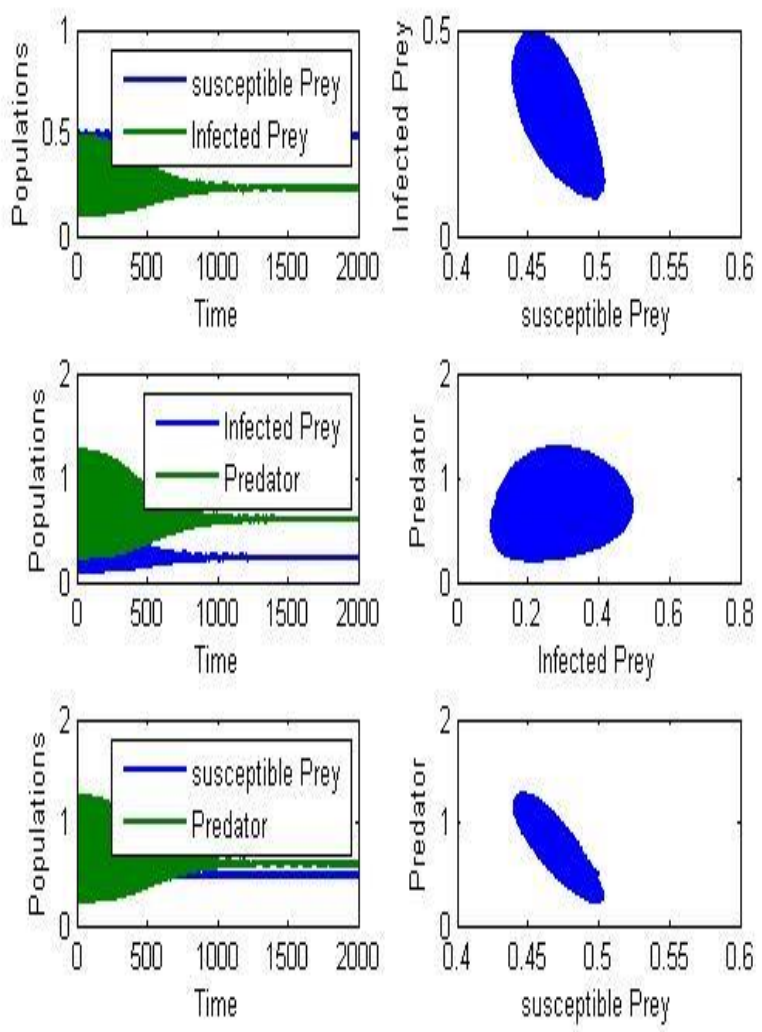

(c)

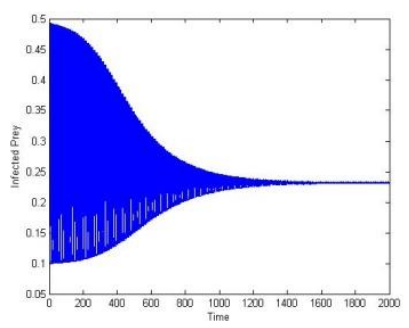

(d)

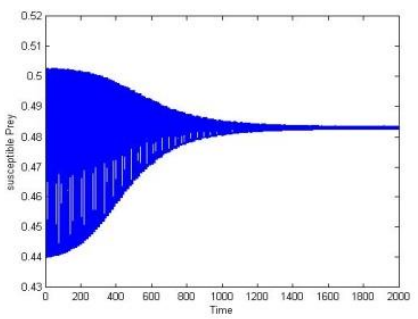

(e)

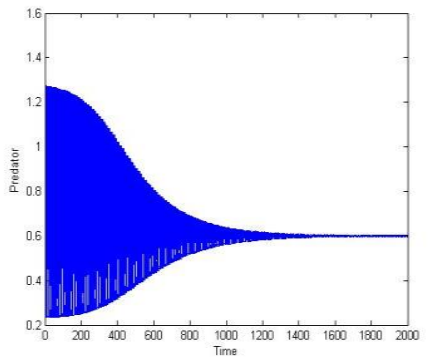

(f)

Figure 5.3:(a) a time profile of the steady state with respect to the populations of x, y, z . (b) phase portrait at $\stackrel{*}{E}=(0.5411$, $0.1689,0.5680$ ) with the parameter values $m_{0}=0.000918$; $\alpha_{1}=6.019 ; \alpha_{3}=0.1099 ; \alpha_{4}=0.00107 ; \alpha_{5}=0.104 ; \alpha_{7}=$ $0.79 ; \alpha_{8}=3.990 ; k_{1}=1.92 ; k_{2}=5 ; k_{3}=1.114$. (c) a time profile and phase portraits in two dimensional plane for the above parameter values. (d) a time profile of Infected prey population. (e) a time profile of Susceptible prey population. (d) a time profile of Predator population.

\section{HOPF BIFURCATION}

In the present study, various parameters have been used to exhibit the behavior of dynamical system. Eco-Epidemiological models with constant parameters are frequently found to approach a steady state where species coexist in equilibrium. The behavior of a system may change in relation to the parameters used in the model. Such parameters which cause the transition in a system are named as bifurcation points. At any point where the system has nontrivial periodic solutions, a Hopf bifurcation occurs.

The following theorem established that Hopf bifurcation occurs for the system (2.2) at a sensitive value $\alpha_{7}=\alpha_{7}{ }^{*}$. For proving this, we follow Liu [6,27] approach. 
Theorem(6.1): At $\alpha_{7}=\alpha_{7}{ }^{*}$ the model (2.2) occurs Hopf bifurcation along with the local stability conditions (i),(ii),(iii) of theorem (5.1) holds.

Proof:

let $\alpha_{7}^{*}=\frac{y^{*}\left[\begin{array}{l}F^{*} G^{*} z^{*}-N_{2} \alpha_{8} F^{*} N_{3} z^{*}+\alpha_{5} H^{*} G^{*} N_{2}{ }^{4} N_{3}{ }^{2} \\ +\alpha_{8} N_{2}{ }^{2} N_{3}{ }^{2} N_{1} z^{*}\left(G^{*} N_{1}^{2}+\alpha_{3} \alpha_{5} x^{*} N_{3} N_{2}^{3}\right)\end{array}\right]}{x^{*} z^{*}\left(\begin{array}{l}N_{2}{ }^{4} N_{3} N_{1}^{2} \\ \left(m_{0}-x^{* 2}\right)\end{array}\right)\left[\begin{array}{l}\left(\alpha_{3} N_{1} y^{*} z^{*}\right) \\ \left(1-N_{3}{ }^{2}\right)-\alpha_{3} N_{1} F^{*}+H^{*} N_{2} N_{3} y^{*}\end{array}\right]}$.

Where $G^{*}=F^{*}+y^{*} z^{*} N_{3}^{2} ; H^{*}=x-\alpha_{4} N_{1}$, Then

$\left.B_{1}\right|_{\alpha_{7}=\alpha_{7}^{*}}=\frac{y^{*}}{N_{2}^{2}}\left(1+z^{*}\right)+\alpha_{3} z^{*}\left(m_{0}-x^{* 2}\right)\left(m_{0}+x^{* 2}\right)^{-2}+\alpha_{1}\left(2 k_{1} x^{*}-1\right)>0$

$\left.B_{3}\right|_{\alpha_{7}=\alpha_{7}^{*}}=\frac{\alpha_{8} y^{*} z^{*}}{N_{2}^{5} N_{3}^{2}}\left[y^{*} N_{3}^{2}+\alpha_{3} z^{*}\left(m_{0}-x^{* 2}\right) N_{2}^{2}+\alpha_{1}\left(2 k_{1} x^{*}-1\right) N_{2}^{2} N_{3}^{2}\right]$

$$
-\frac{\alpha_{3} y^{*} x^{*} z^{*}}{N_{1}^{2} N_{2}^{2} N_{3}^{2}} \alpha_{5} \alpha_{8} N_{3}+\left\{\left[\frac{y^{*} z^{*}\left(m_{0}-x^{* 2}\right)\left(x^{*}-\alpha_{4} N_{1}\right)}{N_{1} N_{2} N_{3}^{2}}+\right.\right.
$$

$$
\left.\frac{\alpha_{3} y^{*} x^{*} z^{*}}{N_{1}^{2} N_{2}^{2} N_{3}^{2}}\left(\frac{z^{*}\left(m_{0}-x^{* 2}\right) N_{1}^{2}}{N_{3}}\right)\right] \text {. }
$$$$
\left.\left[\frac{y^{*}\left[\begin{array}{l}
F^{*} G^{*} z^{*}-N_{2} \alpha_{8} F^{*} N_{3}{ }^{2} z^{*}+\alpha_{5} H^{*} G^{*} N_{2}{ }^{4} N_{3}{ }^{2} \\
+\alpha_{8} N_{2}{ }^{2} N_{3}{ }^{2} N_{1} z^{*}\left(G^{*} N_{1}{ }^{2}+\alpha_{3} \alpha_{5} x^{*} N_{3} N_{2}{ }^{3}\right)
\end{array}\right]}{x^{*} z^{*}\left(N_{2}{ }^{4} N_{3} N_{1}{ }^{2}\left(m_{0}-x^{* 2}\right)\right)\left[\left(\alpha_{3} N_{1} y^{*} z^{*}\right)\left(1-N_{3}{ }^{2}\right)-\alpha_{3} N_{1} F^{*}+H^{*} N_{2} N_{3} y^{*}\right]}\right]\right]>0 .
$$

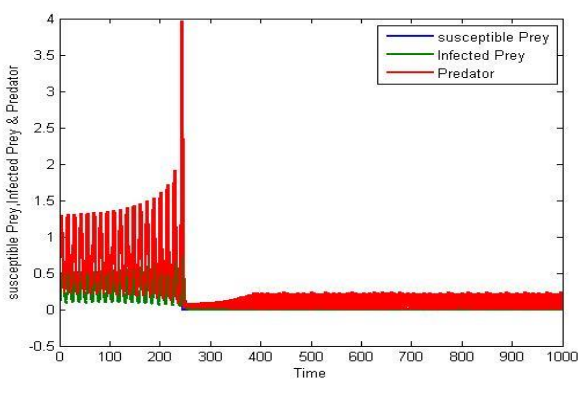

(a)

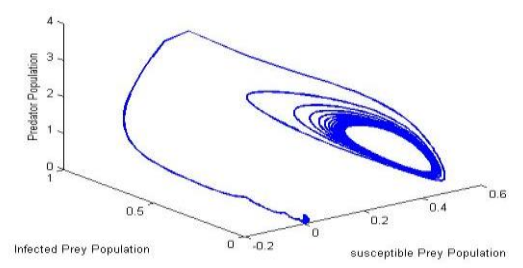

(b)
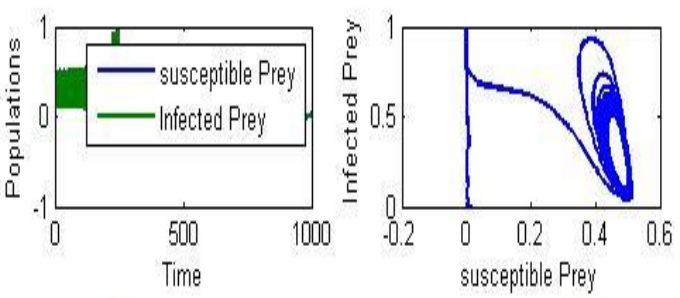

Therefore, $\left.\frac{d \Delta}{d \lambda}\right|_{\alpha_{7}=\alpha_{7}^{*}} \neq 0$. Hence, a simple Hopf bifurcation occurs at $\alpha_{7}=\alpha_{7}{ }^{*}$.

\section{Numerical Simulations:}

Using the same set values in Figure 5.3, from the theorem (6.1), we can determine the critical value of $\alpha_{7}$ and it is $\left(\alpha_{7}^{*}\right)=0.79$. The system is unstable for $\alpha_{7}>\alpha_{7}^{*}$ around the positive equilibrium point $\stackrel{*}{E}$, taking $\alpha_{7}{ }^{*}=0.81$ the solution of the system (2.2) has been shown in Fig: 6.1(b) , which indicate that the system is unstable around the positive
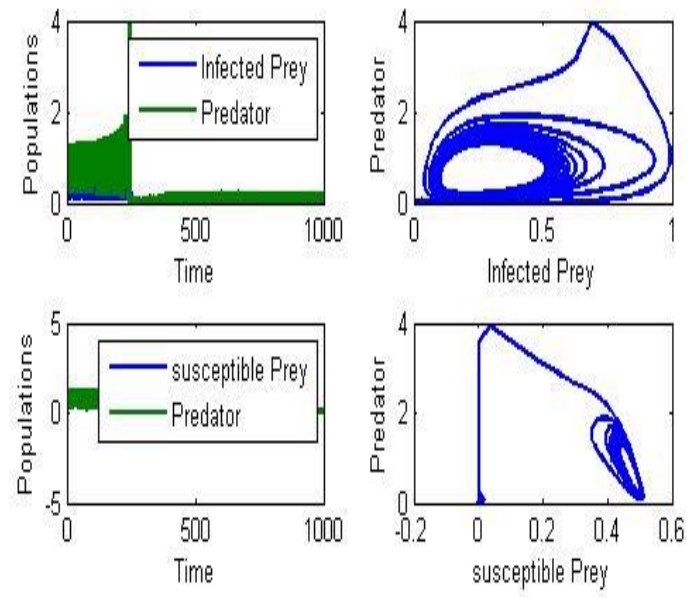

(c) 


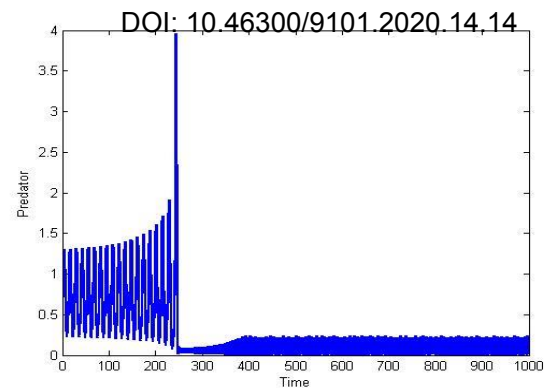

(d)

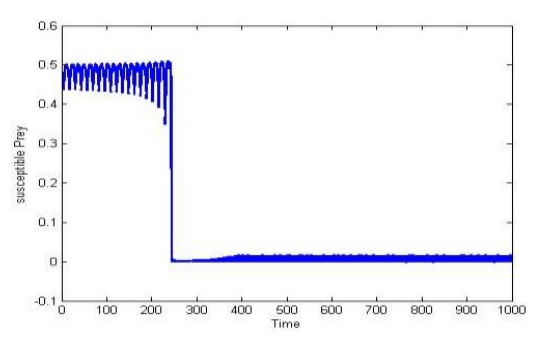

(e)

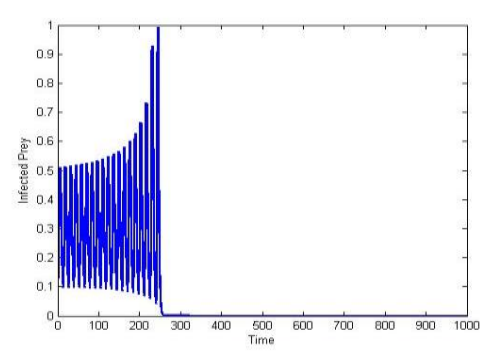

(f)

Figure 6.1:(a) a time profile of the steady state with respect to the populations of $\mathrm{x}, \mathrm{y}, \mathrm{z}$. (b) Bifurcation diagram for $\alpha_{7}=0.81$. (c) a time profile and phase portraits in two dimensional unstable graphs. (d) a time profile of Predator population. (e) a time profile of Susceptible prey population. (f) a time profile of Infected prey population.

\section{Stochastic analysis}

In this section, the stochastic version of the model has been formulated by considering the influence of the random noise which is in the form of additive Gaussian white noise to the model (2.2) and the perturbations are as fallows

$$
\begin{aligned}
& \frac{d x}{d t}=\left(\alpha_{1} x-\alpha_{1} k_{1} x^{2}\right)-\frac{x y}{1+x}-\alpha_{2} \frac{x z}{m_{0}+x^{2}}+\alpha_{4} y+p_{4} \cdot \eta_{4}(t) \\
& \frac{d y}{d t}=\alpha_{5} y\left(\frac{x}{1+x}-k_{2}\right)+\frac{y z}{1+y}+p_{2} \cdot \eta_{2}(t) \\
& \frac{d z}{d t}=\alpha_{7} y\left(\frac{x}{m_{0}+x^{2}}-k_{3}\right)-\alpha_{8} \frac{y z}{1+y}+p_{2} \cdot \eta_{3}(t)
\end{aligned}
$$

Where $p_{1}, p_{2}, p_{3}$ are constants and $\eta(t)=\left(\eta_{i}(t)\right)$ is a $3 \mathrm{D}$ Gaussian White noise parameters satisfying $\mathrm{E}\left[\eta_{i}(t)\right]=0 ;$ where $i=1,2,3$.

Let $\quad x=\mu_{1}+S^{*} ; y=\mu_{2}+R^{*} ; z=\mu_{3}+\mathrm{T}^{*}$; then $\dot{x}=\dot{\mu}_{1} ; \dot{y}=\dot{\mu}_{2} ; \dot{z}=\dot{\mu}_{3} ;$ where $\dot{\mu}_{i}=\frac{d \mu_{i}}{d t} ; i=1,2.3$.

By neglecting higher power of $\eta_{i}(t)$, equation (7.1) becomes

$$
\begin{aligned}
& \dot{\mu_{1}(t)}=-2 k_{1} \alpha_{1} \mu_{1} S^{*}-\mu_{2} S^{*}-\frac{\alpha_{3}}{m_{0}} \mu_{3} S^{*}+p_{1} \eta_{1} ; \\
& \dot{\mu_{2}(t)}=\alpha_{5} \mu_{1} R^{*}+\mu_{3} R^{*}+p_{2} \eta_{2} ;
\end{aligned}
$$

$$
\dot{\mu_{3}}(t)=\frac{\alpha_{7}}{m_{0}} \mu_{1} \mathrm{~T}^{*}-\alpha_{8} \mu_{2} \mathrm{~T}^{*}+p_{3} \eta_{3} ;
$$

Applying Fourier Transform on both sides of (7.1.1), we get

$$
p_{1} \bar{\eta}_{1}(\omega)=\left(i \omega+2 \alpha_{1} k_{1} S^{*}\right) \overline{\mu_{1}}(\omega)+\bar{\mu}_{2}(\omega) S^{*}+\frac{\alpha_{3}}{m_{0}} \bar{\mu}_{3}(\omega) S^{*}
$$

$p_{2} \bar{\eta}_{2}(\omega)=-\alpha_{5} \bar{\mu}_{1}(\omega) R^{*}+i \omega \cdot \overline{\mu_{2}}(\omega)-\overline{\mu_{3}}(\omega) R^{*}$

$p_{3} \bar{\eta}_{3}(\omega)=-\frac{\alpha_{7}}{m_{0}} \overline{\mu_{1}}(\omega) \mathrm{T}^{*}+\alpha_{8} \bar{\mu}_{2}(\omega) \mathrm{T}^{*}+i \omega \cdot \bar{\mu}_{3}(\omega)$

$\mathrm{M}(\omega) \bar{\mu}(\omega)=\bar{\eta}(\omega)$ represents the matrix form of above equations, (7.2)

where 


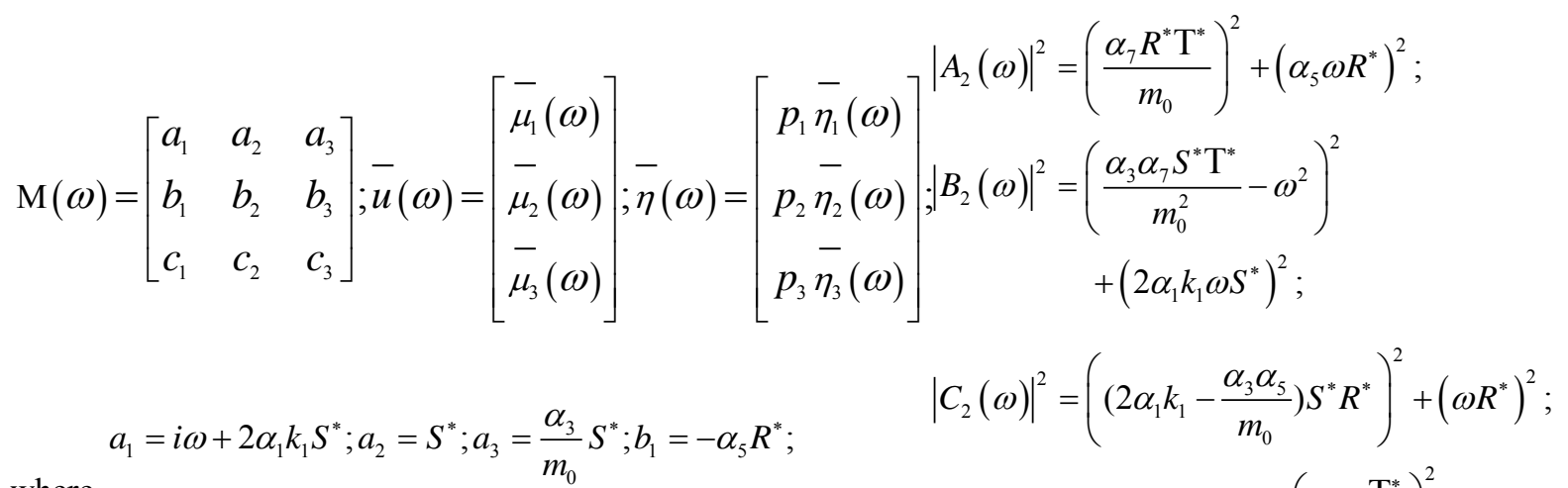
where

$$
b_{2}=i \omega ; b_{3}=-R^{*} ; c_{1}=-\frac{\alpha_{7}}{m_{0}} \mathrm{~T}^{*} ; c_{2}=\alpha_{8} \mathrm{~T}^{*} ; c_{3}=i \omega .
$$

$$
\left|A_{3}(\omega)\right|^{2}=\left(\alpha_{5} \alpha_{8} R^{*} \mathrm{~T}^{*}\right)^{2}+\left(\frac{\alpha_{7} \omega \mathrm{T}^{*}}{m_{0}}\right)^{2}
$$

The above equation (7.2) can also be written as

$$
\bar{\mu}(\omega)=[\mathrm{M}(\omega)]^{-1} \bar{\eta}(\omega)
$$

$$
\begin{aligned}
& \left|B_{3}(\omega)\right|^{2}=\left(\left(2 \alpha_{1} \alpha_{8} k_{1}+\frac{\alpha_{7}}{m_{0}}\right) S^{*} \mathrm{~T}^{*}\right)^{2}+\left(\alpha_{8} \omega \mathrm{T}^{*}\right)^{2} ; \\
& \left|C_{3}(\omega)\right|^{2}=\left(\alpha_{5} R^{*} S^{*}-\omega^{2}\right)^{2}+\left(2 \alpha_{1} k_{1} \omega S^{*}\right)^{2}
\end{aligned}
$$

Let $\quad[\mathrm{M}(\omega)]^{-1}=L(\omega)$, Therefore, $\bar{\mu}(\omega)=L(\omega) \bar{\eta}(\omega)$

Where $L(\omega)=\frac{\operatorname{Ads}(\mathrm{M}(\omega))}{|\mathrm{M}(\omega)|}$

$$
\begin{aligned}
& X_{1}=\left(\alpha_{8} R^{*} T^{*}-\omega^{2}\right), Y_{1}=0 ; X_{2}=\left(\frac{\alpha_{3} \alpha_{8} S^{*} T^{*}}{m_{0}}\right), Y_{2}=\left(\omega S^{*}\right) ; \\
& X_{3}=\left(S^{*} R^{*}\right), Y_{3}=\left(\frac{\alpha_{3} \omega S^{*}}{m_{0}}\right) ;
\end{aligned}
$$

By considering ( from 8.10,8.11 and 8.12. of [27] )

$\overline{\mu_{i}}(\omega)=\sum_{j=1}^{3} L_{i j}(\omega) \bar{\eta}_{j}(\omega)$ and $S_{\mu_{i}}(\omega)=\sum_{j=1}^{3} \alpha_{j}(\omega)\left|L_{i j}(\omega)\right|^{2} ;$ wher $i=1, X^{4}, \overline{3}=\left(\frac{\alpha_{7} R^{*} T^{*}}{m_{0}}\right), Y_{4}=\left(\alpha_{5} \omega R^{*}\right) ; X_{5}=\left(\frac{\alpha_{3} \alpha_{7} S^{*} T^{*}}{m_{0}^{2}}-\omega^{2}\right)$, then we obtain

$$
Y_{5}=\left(2 \alpha_{1} k_{1} \omega S^{*}\right) ; X_{6}=\left(\left(2 \alpha_{1} k_{1}-\frac{\alpha_{3} \alpha_{5}}{m_{0}}\right) S^{*} R^{*}\right), Y_{6}=\left(\omega R^{*}\right) ;
$$

$\sigma_{\mu_{i}}^{2}=\frac{1}{2 \pi}\left\{\begin{array}{l}\int_{-\infty}^{\infty} p_{1}\left|\frac{A_{i}}{|\mathrm{M}(\omega)|}\right|^{2} d \omega+\int_{-\infty}^{\infty} p_{2}\left|\frac{B_{i}}{|\mathrm{M}(\omega)|}\right|^{2} d \omega \\ +\int_{-\infty}^{\infty} p_{3}\left|\frac{C_{i}}{|\mathrm{M}(\omega)|}\right|^{2} d \omega\end{array}\right\} i=1,2,3$

$X_{7}=\left(\alpha_{5} \alpha_{8} R^{*} \mathrm{~T}^{*}\right), Y_{7}=\left(\frac{\alpha_{7} \omega \mathrm{T}^{*}}{m_{0}}\right) ; X_{8}=\left(\left(2 \alpha_{1} \alpha_{8} k_{1}+\frac{\alpha_{7}}{m_{0}}\right) S^{*} \mathrm{~T}^{*}\right)$,

(7. $\hat{P}_{8}^{)}=\left(\alpha_{8} \omega \mathrm{T}^{*}\right) ; X_{9}=\left(\alpha_{5} R^{*} S^{*}-\omega^{2}\right), Y_{9}=\left(2 \alpha_{1} k_{1} \omega S^{*}\right)$.

By substituting above values in (7.3), then

where $\quad|\mathrm{M}(\omega)|=R(\omega)+i(\operatorname{Im} g(\omega))$ here

$$
\begin{aligned}
|\mathrm{M}(\omega)|= & {\left[\frac{1}{m_{0}}\left(2 \alpha_{1} k_{1} m_{0}+\alpha_{7}-\alpha_{3} \alpha_{5} \alpha_{8}\right) S^{*} R^{*} \mathrm{~T}^{*}-2 \alpha_{1} k_{1} \omega^{2} S^{*}\right] } \\
+i & {\left[\frac{\alpha_{3} \alpha_{7} \omega}{m_{0}^{2}} S^{*} \mathrm{~T}^{*}+\alpha_{8} \omega R^{*} \mathrm{~T}^{*}+\alpha_{5} \omega S^{*} R^{*}-\omega^{3}\right] } \\
\left|A_{1}(\omega)\right|^{2}= & \left(\alpha_{8} R^{*} R^{*}-\omega^{2}\right)^{2} ;\left|B_{1}(\omega)\right|^{2}=\left(\frac{\alpha_{3} \alpha_{8} S^{*} \mathrm{~T}^{*}}{m_{0}}\right)^{2} \\
& +\left(\omega^{2} S^{* 2}\right) ;\left|C_{1}(\omega)\right|^{2}=\left(S^{*} R^{*}\right)^{2}+\left(\frac{\alpha_{3} \omega S^{*}}{m_{0}}\right)^{2}
\end{aligned}
$$

$$
\sigma_{\mu_{i}}^{2}=\frac{1}{2 \pi}\left\{\int_{-\infty}^{\infty} \frac{1}{R^{2}(\omega)+I^{2}(\omega)}\left[\begin{array}{l}
p_{1}\left\{X_{j_{1}}{ }^{2}+Y_{j_{1}}{ }^{2}\right\} \\
+p_{2}\left\{X_{j_{2}}{ }^{2}+Y_{j_{2}}{ }^{2}\right\} \\
+p_{3}\left\{X_{j_{3}}{ }^{2}+Y_{j_{3}}{ }^{2}\right\}
\end{array}\right]\right\} d \omega,
$$$$
\text { wher }\left\{i=1,2,3 ; j_{1}=1,4,7 ; j_{2}=2,5,8 ; j_{3}=3,6,9\right\} \text {. }
$$

If $p_{1}=0 ; p_{2}=0$, then the population variances are

$$
\sigma_{\mu_{1}}^{2}=\frac{p_{3}\left(X_{3}^{2}+Y_{3}^{2}\right)}{2 \pi} \int_{-\infty}^{\infty} \frac{1}{R^{2}(\omega)+I^{2}(\omega)} d \omega
$$


$\sigma_{\mu_{2}}^{2}=\frac{p_{3}\left(X_{6}^{2}+Y_{6}^{2}\right)}{2 \pi} \int_{-\infty}^{\infty} \frac{1}{R^{2}(\omega)+I^{2}(\omega)} d \omega$

$\sigma_{\mu_{3}}^{2}=\frac{p_{3}\left(X_{9}{ }^{2}+Y_{9}^{2}\right)}{2 \pi} \int_{-\infty}^{\infty} \frac{1}{R^{2}(\omega)+I^{2}(\omega)} d \omega$

If $p_{2}=0, p_{3}=0$, then the population variances are

$\sigma_{\mu_{1}}^{2}=\frac{p_{1}\left(X_{1}^{2}+Y_{1}^{2}\right)}{2 \pi} \int_{-\infty}^{\infty} \frac{1}{R^{2}(\omega)+I^{2}(\omega)} d \omega ;$

$\sigma_{\mu_{2}}^{2}=\frac{p_{1}\left(X_{4}{ }^{2}+Y_{4}^{2}\right)}{2 \pi} \int_{-\infty}^{\infty} \frac{1}{R^{2}(\omega)+I^{2}(\omega)} d \omega ;$

$\sigma_{\mu_{3}}^{2}=\frac{p_{1}\left(X_{7}^{2}+Y_{7}^{2}\right)}{2 \pi} \int_{-\infty}^{\infty} \frac{1}{R^{2}(\omega)+I^{2}(\omega)} d \omega$.

If $p_{1}=0, p_{3}=0$ then the population variances are $\sigma_{\mu_{1}}^{2}=p_{2} \frac{\left(X_{2}^{2}+Y_{2}^{2}\right)}{2 \pi} \int_{-\infty}^{\infty} \frac{1}{R^{2}(\omega)+I^{2}(\omega)} d \omega$

$\sigma_{\mu_{2}}^{2}=p_{2} \frac{\left(X_{5}^{2}+Y_{5}^{2}\right)}{2 \pi} \int_{-\infty}^{\infty} \frac{1}{R^{2}(\omega)+I^{2}(\omega)} d \omega$

$\sigma_{\mu_{3}}^{2}=p_{2} \frac{X_{8}^{2}+Y_{8}^{2}}{2 \pi} \int_{-\infty}^{\infty} \frac{1}{R^{2}(\omega)+I^{2}(\omega)} d \omega$.

Numeric Simulations for stochastic system:

In this section the stochastic model (7.1) is examined numerically in the Figures $7:(a 1, b 1)$ to $7:(a 5, b 5)$ of the varying parameter values $\left(p_{1}, p_{2}, p_{3}\right):(0.1,0.3,0.2)$; $(0.1,0.05,0.7) ;(0.05,0.05,0.17) ;(0.5,0.5,0.19)$;

0.05,0.5.0.9); with the following fixed parameters $m_{0}=$ $0.00009982 ; \alpha_{1}=1.099912 ; \alpha_{3}=0.099872 ; \alpha_{4}=0.099893$ ; $\alpha_{5}=0.199285 ; \alpha_{7}=0.0099 ; \alpha_{8}=0.0999389 ; k_{1}=1.92$; $k_{2}=1.00009 ; k_{3}=1.4091$.

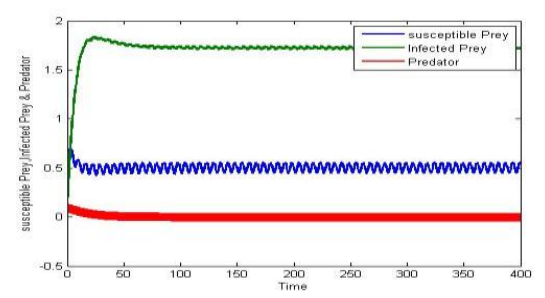

(a1)

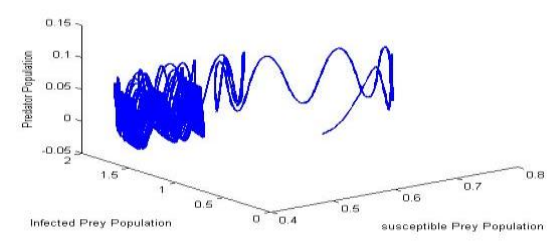

(b1)

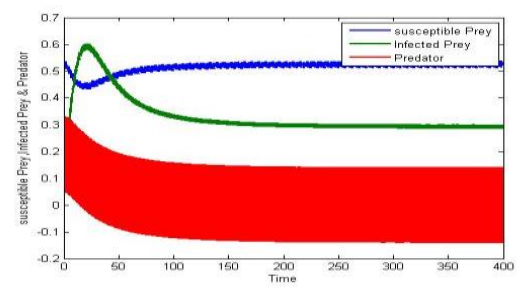

(a2)

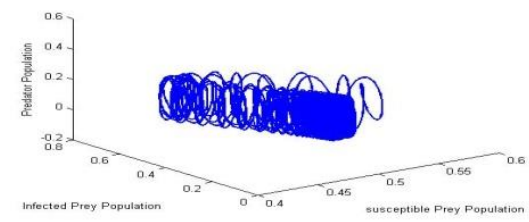

(b2)

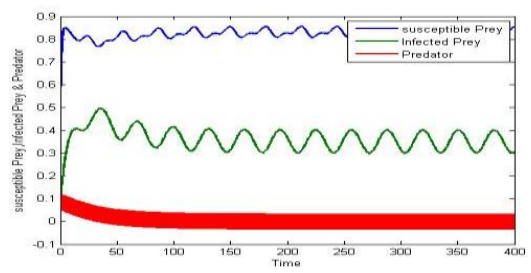

(a3) 


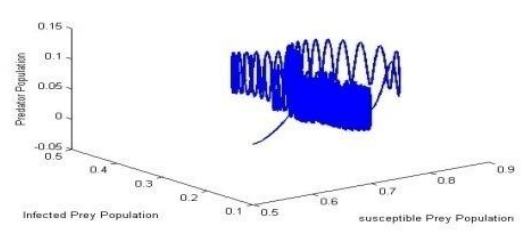

(b3)

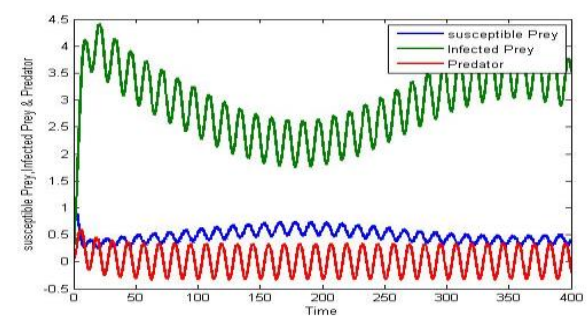

(a4)

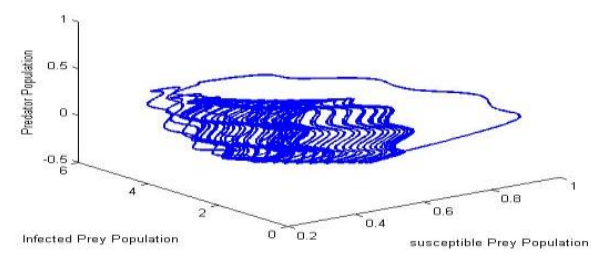

(b4)

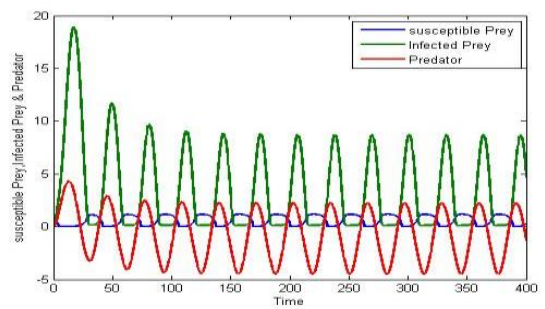

(a5)

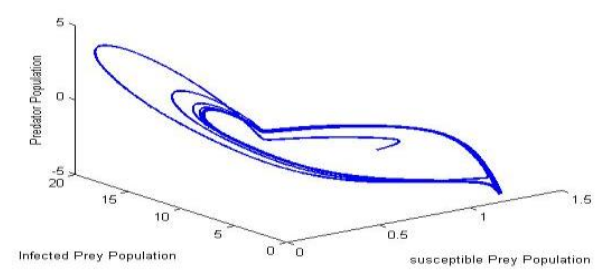

(b5)
Figures $7:(a 1)$ to (a5) represents variation of $\mathrm{x}, \mathrm{y}, \mathrm{z}$ verses time $t$ and Figures 7: (b1) to (b5) represents phase portraits of variation of $\mathrm{x}, \mathrm{y}, \mathrm{z}$ with different $p_{1}, p_{2}, p_{3}$ parameters values.

\section{CONCLUSIONS}

In this paper, we consider the three species Eco-Epidemiology model with Holling Type-IV and Type-II Predator(z) functional response with Susceptible prey(x) and infected Prey (y) respectively to understand the dynamics of the model. The co-existence steady state $\left(x^{*}, y^{*}, z^{*}\right)$ is exist when the rate of infection $\left(l_{0}\right)$ which less than the rate of infected prey individuals to recover and reenter into susceptible prey $(\delta)$ and also

$\left(\alpha^{*}<\delta^{*}<x^{*}<\beta^{*}\right)$ where $\alpha^{*}, \delta^{*}, \beta^{*}$ values are defined in section 4. The stability of the system is carried out locally and globally at the coexistence state. The numerical simulations in section 5 are evident to the stability of the coexistence state point . The global stability at the coexistence state is carried out by constructing the Lyapunov function. It is observed that the proposed model exhibits Hopf bifurcation when The ratio between the growth rate of the predator $(z)$ and the product of the rate of infection $\left(l_{0}\right)$ and the half saturation constant $(\gamma)$ is greater than 0.79 which is equal to $\alpha_{7}$.

we proposed a stochastic version of the model in section 7 and studied the behavior of the stochastic system around the co-existence steady state. From this study we observed that the sensivity of parameters (low and high intensively) causes large environmental fluctuations which leads to chaotic behavior, this can be showed by the figures $7:(a 1, b 1)$ to $7:(a 5, b 5)$ of section 7. So, we conclude that the environmental fluctuations also effect the our EcoEpidemiology model..

\section{REMARKS}

Initially Lotka - Volterra proposed a linear functional response for a pry-predator model and which is unbounded. This response is called Type-I functional response. But, while studying the complexity in model ecosystems need reasonable functional responses that should be nonlinear and bounded.

The predator functional response $\frac{w x}{c+x}$ which is called

Holling Type-II functional response. This functional response describes the predator per capita rate of predation is limited by its capacity to process food. The functional response $\frac{w x}{d+x^{2}}$ is a Holling type-IV functional response. This response function describes a situation in which the predator's per capita rate of predation decreases at sufficiently high prey densities. In this paper we proposed Type-II and Type-IV functional response, which are $\frac{l_{0} x}{\alpha+x}, \frac{l_{3} x}{\beta+x^{2}}$ respectively. By considering these functional responses the model becomes more complex with the more number of parameters. Due to this a third order 
characteristic equation is obtained at the co-existence steady state with the co-efficient having power 4 which is challenging to analyze by using Routh-Horwirtz criteriaReferences.

\section{REFERENCES}

[1] Holling.C.S, "The functional response of predator to prey density and its role in mimicry and population regulation",Mem.Ent.Can.,45(1965),1-60.

[2] Alan Hastings ,"Thomas Powell, Chaos in a ThreeSpecies Food Chain", Ecological Society of America, Vol. 72, (1991), No. 3 pp. 896-903.

[3] Debasis.M, "Bifurcation and Stability Analysis of a Preypredator System with a Reserved Area", World Journal of Modelling and Simulation,Vol.8(2012),No.4,pp.285292.

[4] Freedman.H, "Wolkowicz.G, Predator-prey systems with group defense", The paradox of enrichment revisited Bulletin of Mathematical Biology(1986)., 48:493-508.

[5] K. Das, N. H. Gazi,"Structural Stability Analysis of an Algal Bloom Mathematical Model in Trophic Interaction", International Journal of Non-linear Analysis Real World Applications., Vol. 11(2010), No. 4,pp.21912206.

[6] Liu,W.M,"Criterion of Hopf bifurcation without using Eigen values", j. Math. Anal.Appl.182(1994),250.

[7] Leah Edelstein-keshet,"Mathematical Models in Biology", Society for Industrial and Applied Mathematics.

[8] Jicai Huang, Xiaojing Xia, Xinan Zhang, "Bifurcation of Co dimension 3 in a Predator-Prey System of Leslie Type with Simplified Holling Type IV Functional Response", International Journal of Bifurcation and Chaos,Vol. 26. No. 02(2016), 1650034 .

[9] Mukhopadhyay.B, Battacharyya.R.,"On a three-tier ecological food chain model with deterministic and random harvesting, A mathematical study Nonlinear Analysis Modelling and Control",Vol.16,No.1(2011),7788.

[10] Murray.J.D,"Mathematical Biology II" , Spatial Models and Biomedical Applications, Third Edition, Springer.

[11] Panja.P., Mondal.S.K., "Stability analysis of coexistence of three species prey-predator mode", Nonlinear Dyn, (2016).

[12] Phani Kumar.N, Pattabhiramacharyulu,.N. Ch. ,"A Three Species Ecosystem Consisting of a Prey, Predator and a Host Commensal to the Prey", Int. J. Open Problems Compt. Math., Vol. 3 (March 2010).

[13] Ranjith Kumar Upadhyay, Sharada NandanRaw, " Complexdyanamics of a three species food-chain model with Holling type IV functional response" ,Nonlinear Analysis Modeling and Control, vol.16.No.3(2011).353374.

[14] PhaniKumar.N," Global stability of a commensal- host ecological model with limited resources and both are harvesting at a constant rate", Journal of Experimental Sciences 3(2) (2012), 49-52.

[15] R.M. Anderso, R.M. May, "Infectious Disease of Humans, Dynamics and Control ", Oxford univress,
London(1991).

[16] Shuwen.Z , Lansun.C , "AHolling type-II functional response food chain model with impulsive perturbations ,chaos, solutions and fractals", 24(2005),1269-1278.

[17] Strogatz H.S,"Nonlinear Dynamics and Chaos with applications to Physics ,Biology, Chemistry, and Engineering", Perseus Books.

[18] Wiggins.S, "Introduction to applied nonlinear dynamical systems and chaos",( 1990).

[19] KN Murty, V Balaram, K Viswanadh, " Solution of Kronecker Product Initial Value Problems Associated with First Order Difference System via Tensor-based Hardness of the Shortest Vector Problem", Electronic Modeling V.30(6), pp 19-33(2008)

[20] R.M. May, "Stability in randomly fluctuating deterministic environment",Am.Nat.107(1973,) 621-650.

[21] J.Ripa, P.Lundburg, V.Kaitala, "A general theory of environment noise in ecological food webs", Am,Nat.( 1998) 107256-263.

[22] J. Chattopadhyay, O. Arino, "A predator-prey model with disease in the prey",Nonlinear Anal., 36 (1999), pp. 749-766

[23] Xin-You Meng, Hai-FengHuo, "Stability and Hopf bifurcation in a three-species system with feedback delays", Nonlinear Dyn , 64(2011):349-364.

[24] Andrew M. Bate · Frank M. Hilker, "Complex Dynamics in an Eco-epidemiological Model", Bull Math Biol, 75:2059-2078(2013).

[25] Zhang, J.F., Li, W.T., Yan. X.P, "Multiple bifurcation in a delayed predator-prey diffusion system with a functional response", Nonlinear Anal. RWA 11(2010), 2708-2725.

[26] Yanni Xiao and Lansun Chen, "Analysis of a Three Species Eco-Epidemiological Model", Journal of

Mathematical Analysis and Applications ,258(2001), 733-754 .

[27] S hariprasad, M A S Srinivas, $N$ Phanikumar, "Mathematical Study of Plant-Herbivore-Carnivore System with Holling Type-II Functional Responses", Jour of Adv Research in Dynamical \& Control Systems, Vol. 11(2019), 04-Special Issue.

[28]Bate, A. M., \& Hilker, F. M., "Predator-prey oscillations can shift when diseases become endemic", J. Theor. Biol., $316,1-8(2013)$.

[29] K.N Murty, K.V.K Viswanadh, P Ramesh, Y Wu," Qualitative properties of a system of differential quations involving Kronecker product of matrices", Nonlinear Studies, an international journal, V.20, pp 459-7(2013).

[30] Santanu Biswas, Sourav Kumar Sasmal, Joydev Chattopadhyay, "A delayed prey-predator system with prey subject to the strong Allee effect and disease", Nonlinear Dyn, Springer,(2016).

[30] Harkaran Singh, Joydip Dhar, Harbax S. hatti,"Dynamics of a prey-generalized predator system with disease in prey and gestation delay for predator", Earth Syst. Environ, Springer,(2016).

[31] Chandan Maji1 Debasis Mukherjee Dipak Kesh, "Deterministic and stochastic analysis of an Eco- Epidemiological model", J Biol Phys, Springer Science Business Media B.V(2017). 
[32] Hilker, F. M., \& Schmitz, K.,"Disease-induced stabilization of predator-prey oscillations", J. Theor.

Biol., 225, 299-306(2008)..

[33] Shufan Wang, Zhihui Ma ,WentingWang, "Dynamical behavior of a generalized eco- epidemiology system with prey refuge", Advances in Difference Equations, Springer,(2018).

[34] Kasi Viswanath V. Kanuri, R. Suryanarayana, K.N. Murty," Existence of $\Psi$-bounded solutions for linear differential systems on time scales", Journal of Mathematics and Computer Science, Vol. 20, Iss 1, pp 1- 13( 2019).

[35] Absos Ali shaik,Harekrishna Das, "Dynamics of an ecoepidemiological system with disease in competitive prey species, journal of Applied Mathematics and Computing", Springer,(2019).
[36] Kasi Viswanath ,V. Kanuri, K.N. Murty, "Three-Point boundary value problems associated with first order matrix difference system-existence and uniqueness via shortest and closest Lattice vector methods ", Journal of Nonlinear Sciences and Applications (J. Nonlinear Sci. Appl.),Vol. 12, Iss 11, pp 720-727( 2019).

[37] Tao Feng, Xinzhu Meng, Lidan Liu and Shujing Gao, "Application of inequalities technique to dynamics analysis of a stochastic eco-epidemiology model", Journal of Inequalities and Applications, Springer,(2016).

[38] Mahmoud Moustafa, Mohd Hafiz Mohd, Ahmad Izani Ismail and Farah Aini Abdullah, "Dynamical analysis of a fractional-order eco-epidemiological model with disease in prey population", Advances in Difference Equations, Springer(2020). Creative Commons Attribution License 4.0
(Attribution 4.0 International, CC BY 4.0)

This article is published under the terms of the Creative Commons Attribution License 4.0

https://creativecommons.org/licenses/by/4.0/deed.en US 